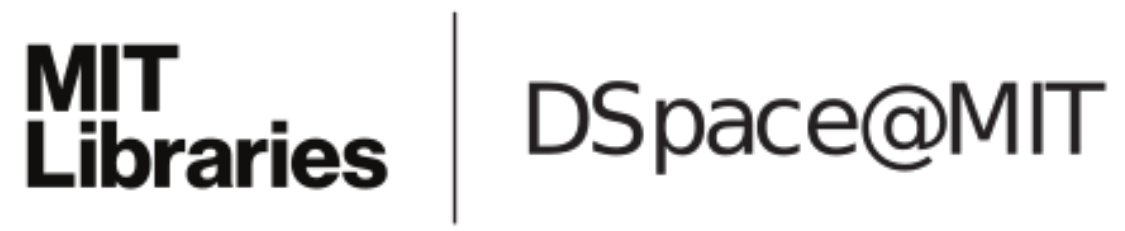

MIT Open Access Articles

Geometry of Molecular Motions in

Dye Monolayers at Various Coverages

The MIT Faculty has made this article openly available. Please share how this access benefits you. Your story matters.

Citation: Vaissier, Valérie, and Troy Van Voorhis. "Geometry of Molecular Motions in Dye Monolayers at Various Coverages." The Journal of Physical Chemistry C 121, 23 (June 2017): 12562-12568 @ 2017 American Chemical Society

As Published: https://pubs.acs.org/doi/10.1021/acs.jpcc.7b02416

Publisher: American Chemical Society (ACS)

Persistent URL: http://hdl.handle.net/1721.1/115102

Version: Author's final manuscript: final author's manuscript post peer review, without publisher's formatting or copy editing

Terms of Use: Article is made available in accordance with the publisher's policy and may be subject to US copyright law. Please refer to the publisher's site for terms of use. 


\title{
Geometry of Molecular Motions in Dye Monolayers at Various Coverages
}

\author{
Valérie Vaissier and Troy Van Voorhis* \\ Department of Chemistry \\ Massachusetts Institute of Technology \\ 77 Massachusetts Avenue \\ Cambridge, MA 02139 \\ E-mail: tvan@mit.edu
}

*To whom correspondence should be addressed 


\begin{abstract}
Molecular motion in monolayers is thought to influence the kinetics of charge transport and recombination in systems such as dye sensitized solar cells (DSSCs). In this work, we use ab-initio molecular dynamics to evaluate the geometry and timescale of such molecular motion in a D102 monolayer. D102 is a dye routinely used in DSSCs, which contains two chemical groups, indoline and triphenylethylene, that are also present in many other dyes. We find that, at low surface coverage, the dye exhibits two main tilting axes around which it heavily distorts within 10 ps. Further, the two benzene rings in the triphenylethylene group rotate with a 3-4 ps period. We observe that these large amplitude movements are suppressed at full coverage, meaning that dyes in a monolayer are locked into place and only undergo minor conformational changes. Our observations indicate that, counter-intuitively, charge diffusion across dye monolayers may be faster in the parts of the system that are characterized by a lower surface coverage. Since charge transport in dye monolayers has been proven to accelerate recombination kinetics in DSSCs, these results provide the basis for a new understanding of the electronic properties of sensitized systems and device efficiency.
\end{abstract}

\title{
Introduction
}

Molecular monolayers have applications in a broad range of research fields from optoelectronics, ${ }^{1-5}$ to catalysis ${ }^{6-8}$ to medicine. ${ }^{9-12}$ They are used in many technologies to tune the fundamental properties of pre-existing materials. In Dye Sensitized Solar Cells (DSSCs) for example, $\mathrm{TiO}_{2}$ nanoparticles are sensitized with a dye molecule monolayer that absorbs a wider range of incoming photons and initiates exciton splitting. ${ }^{13-17}$ Consequently, deriving design rules for optimal dye monolayers is core to the study of DSSCs. These rules are based on a number of characteristics, namely the dye absorption spectrum, the energy level alignment with the metal oxide substrate and the dye adsorption properties, including its binding mode to the surface. ${ }^{18-22}$ Recent theoretical and/or experimental studies have significantly 
contributed to the precise characterization of sensitized $\mathrm{TiO}_{2}$ surfaces (conformational and optical properties) and pushed towards the elaboration of an explicit relationship between the molecular properties of the monolayer and DSSC efficiency. ${ }^{23-31}$ In particular, the arrangement of the dyes within the monolayer (molecular fluctuations, short to medium range order or lack thereof) has been recognized to influence charge injection, recombination and more recently hole transport kinetics. ${ }^{32-36}$ However, more is to be learned about molecular motion in dye sensitized systems, from packing patterns to the geometry and timescale of conformational changes, before a clear link with device characteristics can be established. Characterizing such motions is highly non-trivial, partly due to the organic/inorganic nature of the interface where the dye monolayer sits, which is difficult to probe both experimentally and theoretically. ${ }^{37,38}$ Nevertheless, serious efforts have been made to gain insight into the structural and vibrational dynamics of sensitized metal oxide surfaces. STM, X-Ray, NMR, IR, photoelectron spectroscopy and Molecular Dynamics (MD) allow for an atomistic description of the dye molecule / metal oxide interface with estimations of the surface area per dye, binding modes and molecular orientation of individual dyes relative to the surface. ${ }^{32,34,39-43}$ Non-linear optical methods such as sum frequency generation spectroscopy (SFG) are especially relevant to dye sensitized systems due to their applicability to liquid-solid interfaces and monolayer sensibility. ${ }^{44-49}$ Adsorption structures and orientation distributions of sensitized surfaces are keys to building an accurate picture of DSSCs. ${ }^{50-55}$ Others have investigated the effect of thermal fluctuations on these structural features as well as on the electronic properties of the dyes. ${ }^{33,56-60}$ Nevertheless, up to this date, we do not know how the presence of other dyes affects these orientation distributions, nor whether it changes over time, and if so on what timescale. As a result not many models explicitly account for the effect of molecular motion on charge injection, diffusion and/or recombination in DSSCs. ${ }^{33,61,62}$

Recent Quasi Elastic Neutron Scattering (QENS) investigations show that, at full coverage, anchoring moieties of commonly used dye molecules do not rotate around their anchoring groups on timescales between ten picoseconds and a few nanoseconds. ${ }^{36}$ This suggests that 
changes in conformation of adsorbed dyes occur through part of the molecule that are not directly connected to the anchoring group. Also, the arrangement of dye molecules results from the competition between the adsorption strength to the surface and the steric hindrance within the monolayer. Consequently, we expect the molecular degrees of freedom to depend on the surface coverage with high coverage areas showing series of molecular orientations that differ from low coverage areas, yielding to a high configurational disorder within a monolayer. ${ }^{63}$

In this paper, we study the geometry of the motion of indoline and triphenylethylene groups, building blocks of many commonly used dyes (see Figure 1). ${ }^{64,65}$ We look at the degrees of freedom of these groups in D102, one of the few dyes for which we already know the packing pattern on a (101) $\mathrm{TiO}_{2}$ surface. We find that D102 in isolation (e.g. no immediate neighbors) bends along two main tilting axes and the benzene rings of the triphenylethylene group rotate within a couple of picoseconds. We show that these dynamical molecular rearrangements are severely hindered by the presence of other dyes. At full surface coverage, the dyes adopt disordered but static configurations. This heterogeneity is rationalized to have significant effect on device kinetics.

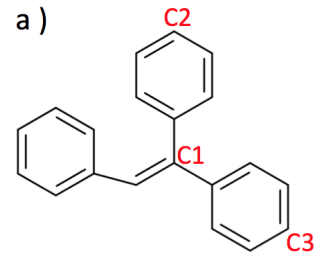

b)

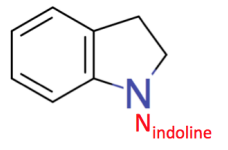

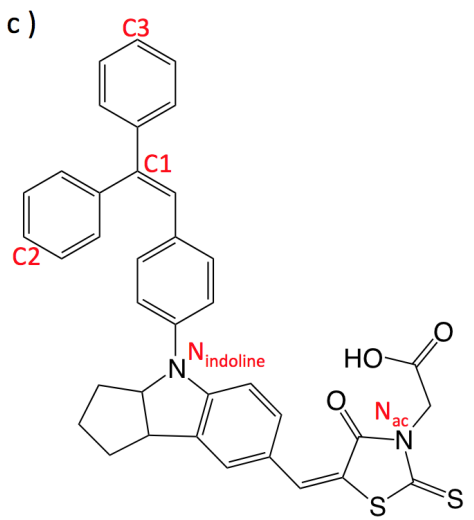

Figure 1. Chemical structure of the molecular building blocks studied in this work. (a) Triphenylethylene (b) indoline and (c) dye D102 with both groups. The red labels mark the atoms whose displacement are considered later in the study. 


\section{Theoretical Methods}

We studied the effect of coverage on the molecular dynamics of a D102 monolayer. We built a periodic lattice using a surface-less unit cell that contains up to four dyes in their energy optimized geometries (mimicking full surface coverage). Although the metal oxide surface is not included in the simulations presented here, the dimensions of the unit cell were chosen to reproduce the preferred positions (bidendate anchoring mode and intermolecular distance) of $\mathrm{D} 102$ on a $\mathrm{TiO}_{2}$ (101) slab (see Figure 2a) along the $\mathrm{x}$ and y directions (the (xy) plane being the plane of the virtual surface) as already published elsewhere. ${ }^{33,34}$ The magnitude of the lattice vectors were $15.14 \AA$ and $20.48 \AA$ in the $\mathrm{x}$ and $\mathrm{y}$ direction respectively, as shown in Figure 2b.
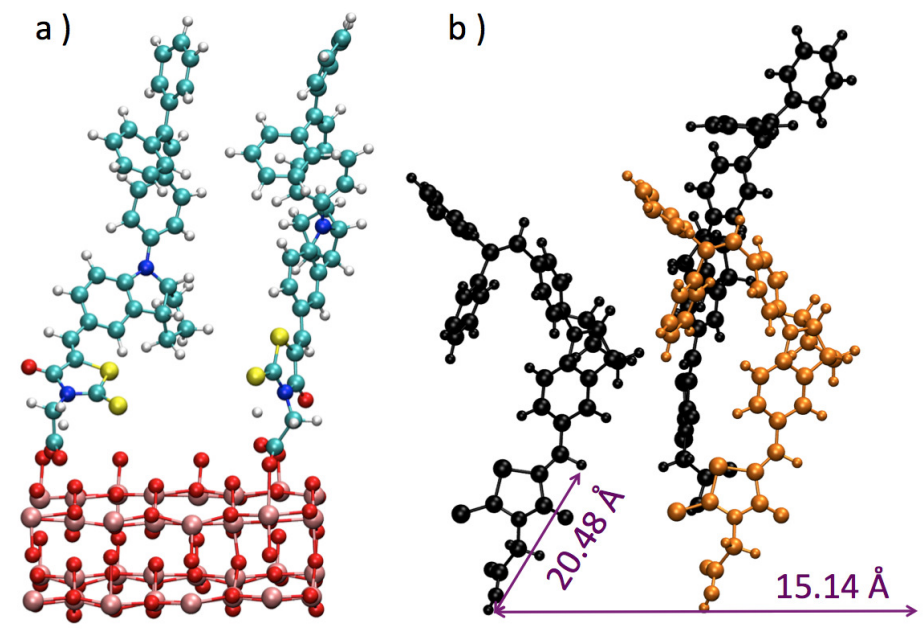

c)

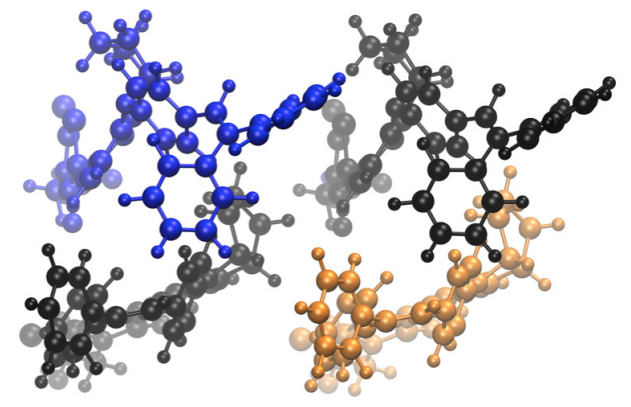

Figure 2. (a) pair of $\mathrm{D} 102$ on a $\mathrm{TiO}_{2}$ slab. The preferred positions of a pair of D102 on $\mathrm{TiO}_{2}$ (101) in both the $\mathrm{x}$ and $\mathrm{y}$ direction were previously found with CPMD simulations. In this work we used these reported preferred positions to build our surface-less unit cell. Atomic color key: red $=$ oxygen, pink $=$ titanium, yellow $=$ sulfur, green $=$ carbon, blue = nitrogen, white = hydrogen. (b) Our unit cell with two D102 diagonally positioned (in black). Also represented the three dye unit cell including the molecule in orange to the previous ones. (c) Top down view of a fully populated unit cell.

We chose a four dye unit cell for two reasons. First, it allows us to simulate multiple surface coverage conditions by removing dyes from the unit cell while preserving its dimension. In this work, we used four, three, two neighboring, two diagonal and one dye per unit 
cell corresponding to $100 \%, 75 \%, 50 \%, 50 \%$ and $25 \%$ surface coverage respectively. Second, it accounts for cross interactions in the unit cells where two dyes are diagonally positioned. For example, we show in Figure $2 \mathrm{~b}$ the unit cell with two dyes in diagonal (in black) and three dyes (adding the orange molecule to the other two). To the best of our knowledge, this is the first time that unit cells with more than a pair of molecules are used for molecular dynamics simulations of dye monolayers.

After energy minimization, we ran 10 ps of Born-Oppenheimer MD (including equilibration) using the software package suite CP2K, ${ }^{66}$ in the NVT ensemble $(300 \mathrm{~K})$ for the five surface coverage conditions described above. We chose ab-initio MD despite its cost because force fields for dye molecules are not readily available. An alternative approach could have been to generate such force field prior to running classical molecular dynamics. However, this would not have been a trivial task and new force fields would need to be developed if the simulations were to be run for other dyes. The striking advantage of the protocol presented here instead is that it can be directly apply to any other system, including co-sensitized surfaces and/or co-adsorbents.

Since we did not include the $\mathrm{TiO}_{2}$ surface in these simulations, we fixed the anchoring group (single carboxylic acid as shown in Figure 1) of each dye to mimic the adsorption to a subjacent substrate (virtual $\mathrm{TiO}_{2}$ surface). By fixing the whole $\mathrm{COOH}$ group, we indirectly make the assumption that the dye molecules bind to the virtual surface in a bidendate mode. To further mimic the presence of the surface, the anchoring group of the dyes were kept protonated, as reported elsewhere. ${ }^{33}$ Note that these conditions do not allow for dye desorption.

The nature of the electrolyte surrounding the dye monolayer in DSSCs differs from device to device. While acetonitrile is the most common solvent, water or solid state materials are also regularly employed. Nevertheless, it is accepted that liquid, as well as solid, electrolytes sit on top of the dye monolayer with only occasional molecules percolating closer to the metal oxide surface. In other words, solvent molecules are not expected to interfere with 
the inter-dye interactions. Consequently, we chose not to incorporate the electrolyte in this work. This keeps our findings general (by being relevant to solid as well as liquid DSSCs) and has the significant advantage to limit the number of variables in our ab-initio model.

Periodic boundary conditions were employed in all directions. As explained above, the magnitude of the lattice vectors were $15.14 \AA$ and $20.48 \AA$ in the $x$ and $y$ direction respectively. In the $z$ direction, we set the magnitude of lattice vector to $40 \AA$ to allow for a vacuum slab spacer of over $10 \AA$. We used Density Functional Theory (DFT) with the combination of $\mathrm{PBE}^{67}$ functional and DZVP ${ }^{68}$ basis set to compute the gradient, as well as a DFTd3 ${ }^{69}$ pair potential correction and a 0.5 fs time step.

\section{Results and Discussion}

In this article, we look at the dynamics of one D102 molecule as we increase the number of its nearest neighbor, up to full surface coverage. With $E_{\text {mon }}$ the total energy of the monolayer, we define the interaction energy, $E_{\text {int }}$ as,

$$
E_{\text {int }}=E_{\text {mon }}-n E_{\text {isolated }}
$$

where $n$ is the number of dyes in the unit cell and $E_{\text {isolated }}$ the energy of D102 in its optimized geometry in gas phase ( $E_{\text {isolated }}$ is therefore a constant). Figure 3 shows the interaction energy $E_{\text {int }}$ (left) and the interaction energy per dye $E_{\text {int }} / n$ (right) for $10 \mathrm{ps}$ NVT trajectories at five surface coverages. The data presented include the equilibration time. Given that there are no free molecules in our system, the equilibration is only necessary to accommodate for the presence of nearest neighbors. This is consistent with the low coverage simulation (black curve) that immediately reaches a stationary state while the high coverage simulation takes the longest to equilibrate (about $2 \mathrm{ps}$ ). For consistency, we exclude the first $2.5 \mathrm{ps}$ in all simulations when calculating averages and standard deviations.

In Table 1, we give the average interaction energy, average interaction energy per dye as 

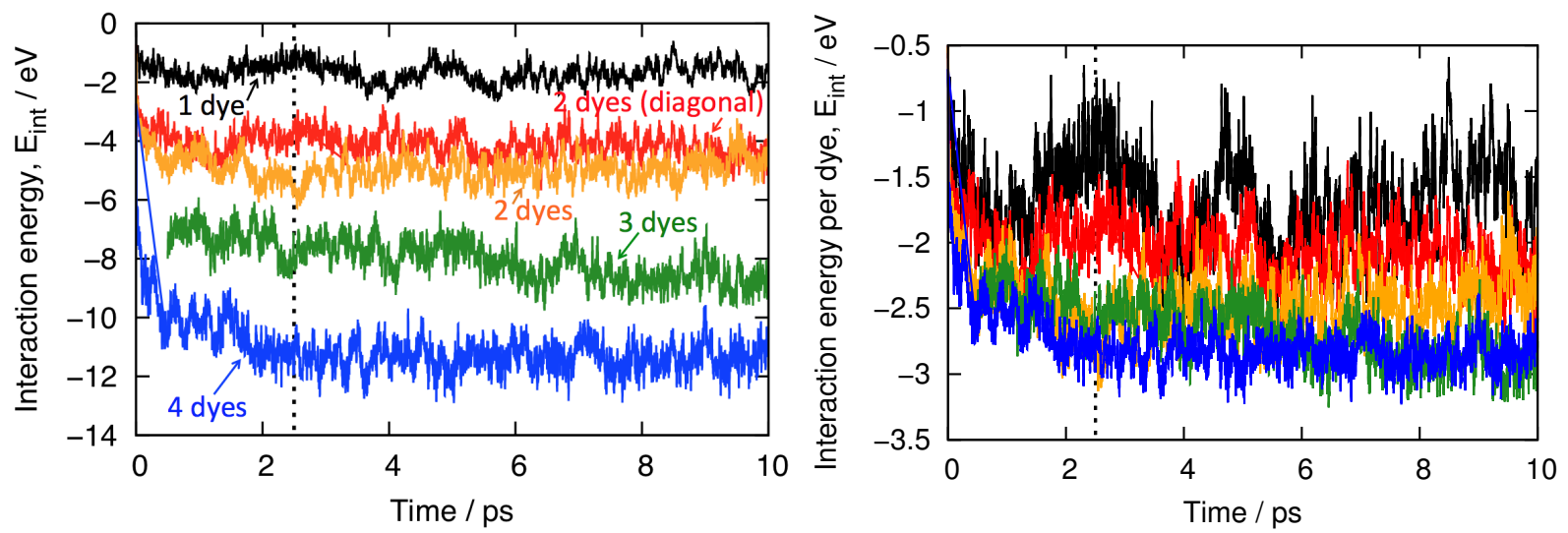

Figure 3. Interaction energy (left) and interaction energy per dye (right) within the dye monolayer at varying surface coverage conditions. The number of dyes per unit cell (which corresponds to a surface coverage) is indicated for each data series (black, red, orange, green and blue is for 1, 2 diagonal, 2, 2 and 4 dyes respectively). The vertical, black dotted line marks the time from which the averages and standard deviations reported in Table 1 are calculated.

well as the standard deviation per molecule for each case. Unsurprisingly, the magnitude of $E_{\text {int }}$ correlates with the coverage condition : the more densely packed the monolayer and the greater the energy difference with respect to isolated molecules. The interaction energy per dye however, plateaus when reaching $75 \%$ coverage. This suggests that the strength of molecular interaction is similar whether a dye is surrounded by 3 or 4 neighbors.

Table 1. Average, average per dye and standard deviation per dye of the interaction energy for the five surface coverage conditions, computed from the data presented in Figure 3.

\begin{tabular}{ccccc}
\hline Surface coverage & $\#$ dyes / unit cell & $\left\langle E_{\text {int }}\right\rangle / \mathrm{eV}$ & $\left\langle E_{\text {int }} / n\right\rangle / \mathrm{eV}$ & $\sigma$ per dye $/ \mathrm{eV}$ \\
\hline $25 \%$ & 1 & -1.66 & -1.66 & 0.30 \\
$50 \%$ & 2 (neighbor) & -5.00 & -2.50 & 0.20 \\
$50 \%$ & 2 (diagonal) & -4.27 & -2.13 & 0.18 \\
$75 \%$ & 3 & -8.58 & -2.86 & 0.15 \\
$100 \%$ & 4 & -11.33 & -2.83 & 0.11 \\
\hline
\end{tabular}

We note that the energy fluctuations per dye molecule (e.g. the standard deviations per dye) decrease with increasing surface coverage. This means that the molecules are constrained by their neighbors in the monolayer and explore a fragment of the configuration space. Overall, fully populating the monolayer will decrease the energy fluctuations per 
dye by $0.20 \mathrm{eV}\left(\sim 8 k_{B} T\right)$., which translates into a strong, coverage dependent, site energy disorder. At last, we see that the energetics of a two diagonal dye unit cell resembles the energetics of a two neighboring dye unit cell better than of a one dye unit cell. This seems to invalidate the assumption commonly made that interactions between two dyes diagonally positioned are negligible. These observations have implications for studies of sensitized systems. Indeed, experimental data is often interpreted within the Gaussian disorder model framework where energy disorder is introduced using a constant $(\sim 0.1 \mathrm{eV})$ standard deviation. A more accurate picture may be to have a local disorder term whose tunable magnitude would account for the variations in surface coverage expected in real systems.

Next, we investigate the geometry of the motion of the dyes in the monolayer and how this motion is affected by the surface coverage. Two scenarios are possible: (i) D102 is a rigid molecule and rearrangements only occur via tilting of the entire dye to accommodate for the steric hindrance caused by its nearest neighbors or (ii) D102 is flexible and neighboring dyes will constrain its naturally occurring conformational changes. We show in Figure 4 the displacement of a few atoms along the body of D102 during the 10 ps simulations at varying surface coverages. We chose to track the two nitrogen atoms (the one directly above the anchoring group that we will label $\mathrm{N}_{\mathrm{ac}}$ and the indoline one, labelled $\mathrm{N}_{\text {indoline}}$ ) and three carbons of the triphenylethylene moieties (labelled $\mathrm{C}_{1}, \mathrm{C}_{2}$ and $\mathrm{C}_{3}$ ).

$\mathrm{N}_{\mathrm{ac}}$ serves as a control; positioned slightly above the fixed $\mathrm{COOH}$ anchoring group, its displacement over the 10 ps trajectory rarely exceeds $1 \AA$ and no distinction can be made between the different coverage conditions. However, it is apparent that the coverage strongly influences the displacement of the other atoms, both in the indoline and triphenylethylene groups. Let us focus on the case where only one dye is present in the unit cell (25\% coverage, in black in Figure 4). After a small rotation over a couple of ps, $\mathrm{N}_{\text {indoline }}$ steadily shifts away from its initial position (final displacement of $4 \AA$ ). This indicates that the dye tilts with respect to its starting configuration. This is substantiated by $\mathrm{C}_{1}$ 's identical behavior, only its final displacement is larger $(9 \AA)$ since it sits further up on D102. However, as shown 

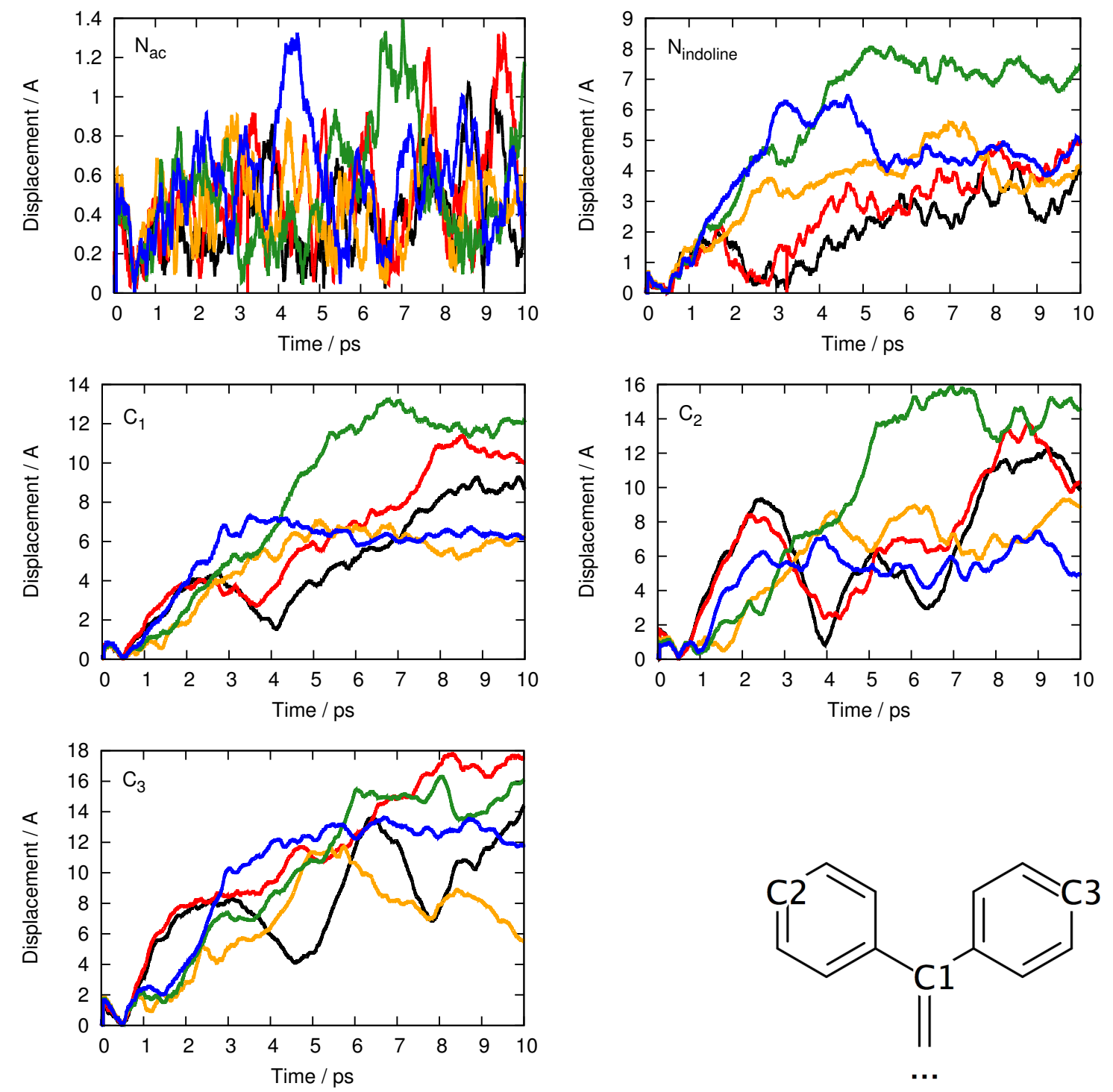

Figure 4. Displacement (absolute value, in $\AA$ ) of the chosen atoms along the body of D102 : $\mathrm{N}_{\mathrm{ac}}, \mathrm{N}_{\text {indoline }}, \mathrm{C}_{1}, \mathrm{C}_{2}$ and $\mathrm{C}_{3}$. The carbons all belong to the triphenylethylene group as shown in the sketch. The displacement was calculated with respect to the position at the simulation starting time. Black $=1$ dye, red $=2$ dyes diagonal, orange $=2$ dyes, green $=3$ dyes, blue $=4$ dyes per unit cell.

by the rather immobile $\mathrm{N}_{\mathrm{ac}}$, this does not seem to be a tilt of the entire dye, rather the triphenylethylene group is tilted with respect to the indoline group which is, in turn, tilted with respect to the rest of the dye as shown in Figure 5a. Meanwhile, the benzene rings on the triphenylethylene group further rotate around the $\left(\mathrm{C}_{1} \mathrm{C}_{2}\right)$ and $\left(\mathrm{C}_{1} \mathrm{C}_{3}\right)$ axes. Although $\mathrm{C}_{2}$ and $\mathrm{C}_{3}$ both rotate a few times within the simulated time, it looks like the motion isn't 
quite symmetric as one may have expected. This is due to the fact that D102 is not standing up on the surface. Rather, it is bent, causing the triphenylethylene group to not align onto the normal to the virtual surface plane, as illustrated in Figure 2. As a result, one of the benzene rings is pointing downwards and is in closer interaction with the rest of the dye, affecting its rotation. In summary, we observe two tilting axes as well as the independent rotation of two benzene rings, meaning that D102 is a flexible molecule naturally undergoing conformational changes (aforementioned scenario (ii)).

Let us now look at the effect of the surface coverage on these large amplitude motions. Overall, the displacement of the atoms for the case with two dyes diagonally positioned in the unit cell (red in in Figure 4) follows quite closely the displacement of the atoms for the case with one dye in the unit cell, except for $\mathrm{C}_{3}$ that no longer rotates. In this case, the other dye prevents the closest benzene ring of the triphenylethylene group from rotating. This is consistent with the absence of $\mathrm{C}_{3}$ rotation when the monolayer is even more populated (three and four dye unit cells in green and blue respectively in Figure 4). When the second dye is next to the dye considered, as opposed to at a diagonal, $\mathrm{C}_{3}$ still rotates but takes twice as long to do so (in orange in Figure 4): the neighboring dye hinders the rotation of the benzene but does not suppress it completely.
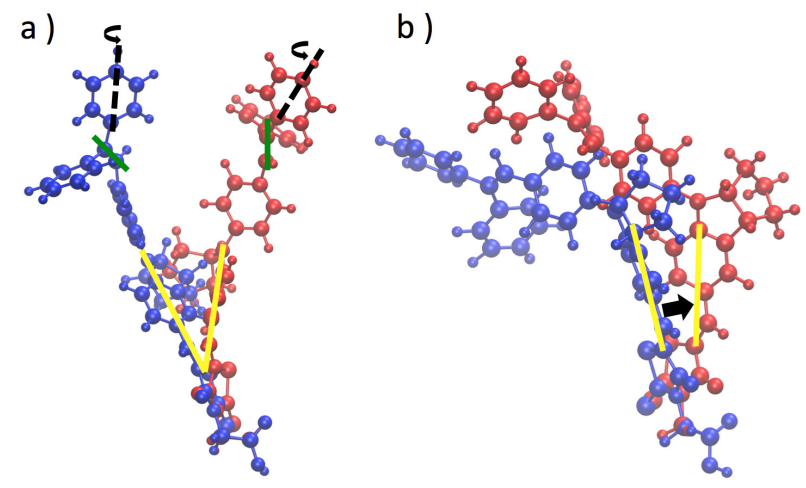

Figure 5. Comparison of snapshots taken at the beginning (blue) and the end (red) of the 10 ps MD simulation at (a) 25\% and (b) 100\% surface coverage. The tilt axes are represented in yellow and green while the rotation axis of the benzene rings are shown in black.

At full coverage condition, the displacement of all the atoms plateau within 4 ps. This 
implies that the dyes tilt slightly and lock one another in place in the monolayer (see Figure 5b), as has been observed in other systems. ${ }^{63}$ This finding also agrees with previous CPMD simulations of dyes on $\mathrm{TiO}_{2}$ (full coverage condition) where no large amplitude motion of the molecules was observed ${ }^{33,34}$ as well as with recent classical MD simulations. ${ }^{70}$ The highest displacements are observed at $75 \%$ coverage (three dye unit cell in green in Figure 4) where the motion is similar to the full coverage case but the dyes tilt few angstroms more for each atoms.

Overall, dynamical molecular rearrangement is only observed at low surface coverage. As the number of molecules per unit area increases, the rotation of dye fragments are repressed and only a rigid displacement of the whole dye, accommodating the steric hindrance, is observed (scenario (ii)). In a recent study, Monti and coworkers ${ }^{70}$ investigated the short-tomedium range order of $\mathrm{D} 102$ on $\mathrm{TiO}_{2}$. They found that, at high coverage, the monolayer is organized in domains; with packing units characterized by a different orientation. They conclude that $\pi$-stacking and T-shaped interactions are the main driving forces toward the formation of ordered surface aggregates. The present study suggests that these forces would be weakened at lower surface coverage because the rigid displacement of the molecules will vary the distance between interacting aromatic rings. As a result, we would expect the domains to be smaller at lower coverage conditions.

These findings have implications for the kinetics of charge transfer and recombination in DSSCs. Indeed, it has been showed that dynamical molecular rearrangement could accelerate transport in dye monolayers by enabling charges to escape configurational traps. ${ }^{33}$ More specifically, ignoring the dynamical rearrangement of the dyes D102 and D149 led to an underestimation of hole diffusion coefficients by at least a factor of 15 . Although these kinetics are still relatively slow (diffusion coefficient of $\sim 10^{-8} \mathrm{~cm}^{2} . \mathrm{s}^{-1}$ compared with $\sim 10^{-6}$ $\mathrm{cm}^{2} \cdot \mathrm{s}^{-1}$ in organic crystals for example), it was shown that charge diffusion in dye monolayers reduces the lifetime of photo-generated charges by up to two orders of magnitude. ${ }^{35}$ In other words, charge transport across the interface accelerates recombination kinetics in 
DSSCs. Therefore, it becomes essential to understand and control the transport properties of sensitized systems. In this work, we show that the fully populated monolayer loses the dynamical signature of the molecular rearrangement of D102 in isolation. In real systems, we expect the monolayer to exhibit a number of low coverage areas (due to local dye desorption for example) within an otherwise fully populated monolayer. This implies that, somewhat counter-intuitively, charge diffusion may be faster in the parts of the dye monolayer that are characterized by a lower surface coverage. This study provides evidence that a clever control of the coverage in dye monolayers could results in a fine tuning of their electronic properties, ultimately reducing recombination losses in DSSCs.

\section{Conclusion}

In this study, we modeled a D102 monolayer at five surface coverages. We showed that at low surface coverage, D102 exhibits large amplitude motions, with tilting indoline and triphenylethylene groups as well as rotating benzene rings. We expect these findings to be transferable to other dye molecules that contain similar chemical groups. We also showed how these motions are suppressed when the coverage increases. In real systems, the dye monolayer is likely to be inhomogeneous with high and low coverage areas. This means that the strong configurational disorder inherent to these systems varies in magnitude and dynamical signature across the monolayer. This study provides the basis for quantifying the effect of molecular dynamics on the kinetics of charge transport and recombination as previously hypothesized. It also invalidates the use of a small constant disorder when analyzing and interpreting experimental data. Rather, a variable disorder, for which inhomogeneity in surface coverage sets inhomogeneity in molecular dynamics, would provide a more realistic

picture of dye sensitized systems. At the simplest level, this could be achieved by using existing models but with an effective disorder term: $\sigma_{\text {eff }}=\alpha \sigma$. The coefficient $\alpha$ would vary from 1 for high coverage to a higher number (3 in this case) for low coverage. $\sigma$ 
would be a term that is dye specific, quantifying the flexibility of the molecule studied. Using such an effective disorder term would allow for more accurate comparison between systems; i.e. keeping $\sigma$ constant but varying $\alpha$ or vice-versa. In the future, we would need to reconcile the various pieces of theory developed so far and build a general model for charge transport and recombination in DSSCs that accounts for a sensible description of energetic and configurational disorder in the dye monolayer.

\section{Acknowledgement}

This work was supported by a grant from the National Science Foundation (CHE-1464804).

\section{References}

(1) Wang, Q. H.; Kalantar-Zadeh, K.; Kis, A.; Coleman, J. N.; Strano, M. S. Electronics and optoelectronics of two-dimensional transition metal dichalcogenides. Nat. Nanotechnol. 2012, 7, 699-712.

(2) Coe, S.; Woo, W.-K.; Bawendi, M.; Bulović, V. Electroluminescence from single monolayers of nanocrystals in molecular organic devices. Nature 2002, 420, 800-803.

(3) Lao, C.; Li, Y.; Wong, C. P.; Wang, Z. L. Enhancing the electrical and optoelectronic performance of nanobelt devices by molecular surface functionalization. Nano Lett. 2007, 7, 1323-1328.

(4) Willner, I.; Doron, A.; Katz, E. Gated molecular and biomolecular optoelectronic systems via photoisomerizable monolayer electrodes. J. Phys. Org. Chem. 1998, 11, 546560.

(5) Willner, I.; Willner, B. Layered molecular optoelectronic assemblies. J. Mater. Chem. 1998, 8, 2543-2556. 
(6) Benitez, I. O.; Bujoli, B.; Camus, L. J.; Lee, C. M.; Odobel, F.; Talham, D. R. Monolayers as models for supported catalysts: zirconium phosphonate films containing manganese(III) porphyrins. J. Am. Chem. Soc. 2002, 124, 4363-4370.

(7) Swalen, J. D.; Allara, D. L.; Andrade, J. D.; Chandross, E. A.; Garoff, S.; Israelachvili, J.; McCarthy, T. J.; Murray, R.; Pease, R. F.; et al., . Molecular monolayers and films. Langmuir 1987, 3, 932-950.

(8) Corma, A. From microporous to mesoporous molecular sieve materials and their use in catalysis. Chem. Rev. 1997, 97, 2273-2420.

(9) Han, G.; Ghosh, P.; Rotello, V. M. Functionalized gold nanoparticles for drug delivery. Nanomedicine 2007, 2, 113-123.

(10) Jain, R. K. Delivery of molecular and cellular medicine to solid tumors. Adv. Drug Deliv. Rev. 2001, 46, $149-168$.

(11) Langer, R.; Tirrell, D. A. Designing materials for biology and medicine. Nature 2004, 428, 487-492.

(12) Dulcey, C. S.; Georger, J. H. J.; al, Deep UV photochemistry of chemisorbed monolayers: patterned coplanar molecular assemblies. Science 1991, 252, 551.

(13) Hagfeldt, A.; Boschloo, G.; Licheng, S.; Kloo, L.; Peterson, H. Dye-sensitized solar cells. Chem. Rev. 2010, 110, 6595-6663.

(14) O’Regan, B.; Grätzel, M. A low-cost, high-efficiency solar cell based on dye-sensitized colloidal $\mathrm{TiO}_{2}$ films. Nature 1991, 353, 737-739.

(15) Grätzel, M. Dye-sensitized solar cells. J. Photochem. Photobiol. C: Photochem. Rev. 2003, 4, $145-153$.

(16) Wei, D. Dye sensitized solar cells. Int. J. Mol. Sci. 2010, 11, 1103-1113. 
(17) Koops, S. E.; ORegan, B. C.; Barnes, P. R. F.; Durrant, J. R. Parameters influencing the efficiency of electron injection in dye-sensitized solar cells. J. Am. Chem. Soc. 2009, 131, 4808-4818.

(18) De Angelis, F.; Fantacci, S.; Mosconi, E.; Nazeeruddin, M. K.; Grtzel, M. Absorption spectra and excited state energy levels of the $\mathrm{N} 719$ dye on $\mathrm{TiO}_{2}$ in dye-sensitized solar cell models. J. Phys. Chem. C 2011, 115, 8825-8831.

(19) Ooyama, Y.; Harima, Y. Molecular designs and syntheses of organic dyes for dyesensitized solar dells. Eur. J. Org. Chem. 2009, 2009, 2903-2934.

(20) Mishra, A.; Fischer, M.; Buerle, P. Metal-free organic dyes for dye-sensitized solar cells: from structure: property relationships to design rules. Angew. Chem. Int. Ed. 2009, 48, 2474-2499.

(21) Hara, K.; Kurashige, M.; Dan-oh, Y.; Kasada, C.; Shinpo, A.; Suga, S.; Sayama, K.; Arakawa, H. Design of new coumarin dyes having thiophene moieties for highly efficient organic-dye-sensitized solar cells. New J. Chem. 2003, 27, 783-785.

(22) De Angelis, F.; Fantacci, S.; Selloni, A. Alignment of the dye's molecular levels with the $\mathrm{TiO}_{2}$ band edges in dye-sensitized solar cells: a DFT-TDDFT study. Nanotechnol. 2008, 19, 424002 .

(23) Xu, M.; Zhang, M.; Pastore, M.; Li, R.; De Angelis, F.; Wang, P. Joint electrical, photophysical and computational studies on D- $\pi$-A dye sensitized solar cells: the impacts of dithiophene rigidification. Chem. Sci. 2012, 3, 976-983.

(24) Kley, C. S.; Dette, C.; Rinke, G.; Patrick, C. E.; ?echal, J.; Jung, S. J.; Baur, M.; Drr, M.; Rauschenbach, S.; Giustino, F. et al. Atomic-scale observation of multiconformational binding and energy level alignment of ruthenium-based photosensitizers on $\mathrm{TiO}_{2}$ anatase. Nano Lett. 2014, 14, 563-569. 
(25) Voïtchovsky, K.; Ashari Astani, N.; Tavernelli, I.; Tetreault, N.; Rothlisberger, U.; Stellacci, F.; Grätzel, M.; Arne Harms, H. In-situ mapping of the molecular arrangement of amphiphilic dye molecules at the $\mathrm{TiO}_{2}$ surface of dye sensitized solar cells. ACS Appl. Mater. Interfaces 2015, 7, 10834-10842.

(26) Sewvandi, G. A.; Chen, C.; Ishii, T.; Kusunose, T.; Tanaka, Y.; Nakanishi, S.; Feng, Q. Interplay between dye coverage and photovoltaic performances of dye-sensitized solar cells based on organic dyes. J. Phys. Chem. C 2014, 118, 20184-20192.

(27) Godlewski, S.; Tekiel, A.; Piskorz, W.; Zasada, F.; Prauzner-Bechcicki, J. S.; Sojka, Z.; Szymonski, M. Supramolecular ordering of PTCDA molecules: the key role of dispersion forces in an unusual transition from physisorbed into chemisorbed state. ACS Nano 2012, 6, 8536-8545.

(28) Sasahara, A.; Fujio, K.; Koide, N.; Han, L.; Onishi, H. STM imaging of a model surface of $\mathrm{Ru}(4,4 \text { ?-dicarboxy-2, 2?-bipyridine })_{2}(\mathrm{NCS})_{2}$ dye-sensitized $\mathrm{TiO}_{2}$ photoelectrodes. Surf. Sci. 2010, 604, 106-110.

(29) Pazoki, M.; Hagfeldt, A.; Boschloo, G. Stark effects in D35-sensitized mesoporous $\mathrm{TiO}_{2}$ : influence of dye coverage and electrolyte composition. Electrochim. Acta 2015, 179, $174-178$.

(30) Hahlin, M.; Johansson, E. M.; Plogmaker, S.; Odelius, M.; Hagberg, D. P.; Sun, L.; Siegbahn, H.; Rensmo, H. Electronic and molecular structures of organic dye/ $/ \mathrm{TiO}_{2}$ interfaces for solar cell applications: a core level photoelectron spectroscopy study. Phys. Chem. Chem. Phys. 2010, 12, 1507-1517.

(31) Pazoki, M.; Lohse, P. W.; Taghavinia, N.; Hagfeldt, A.; Boschloo, G. The effect of dye coverage on the performance of dye-sensitized solar cells with a cobalt-based electrolyte. Phys. Chem. Chem. Phys. 2014, 16, 8503-8508. 
(32) Gebauer, R.; De Angelis, F. A combined molecular dynamics and computational spectroscopy study of a dye-sensitized solar cell. New J. Phys. 2011, 13, 085013.

(33) Vaissier, V.; Mosconi, E.; Moia, D.; Pastore, M.; Frost, J. M.; De Angelis, F.; Barnes, P. R. F.; Nelson, J. Effect of molecular fluctuations on hole diffusion within dye monolayers. Chem. Mater. 2014, 26, 4731-4740.

(34) Pastore, M.; De Angelis, F. Aggregation of organic dyes on $\mathrm{TiO}_{2}$ in dye-sensitized solar cells models: an ab initio Investigation. ACS Nano 2010, 4, 556-562.

(35) Moia, D.; Szumska, A.; Vaissier, V.; Planells, M.; Robertson, N.; ORegan, B. C.; Nelson, J.; Barnes, P. R. F. Interdye hole transport accelerates recombination in dye sensitized mesoporous films. J. Am. Chem. Soc. 2016, 138, 13197-13206.

(36) Vaissier, V.; Sakai, V. G.; Li, X.; Cabral, J. T.; Nelson, J.; Barnes, P. R. F. How mobile are dye adsorbates and acetonitrile molecules on the surface of $\mathrm{TiO}_{2}$ nanoparticles? A quasi-elastic neutron scattering study. Sci. Rep. 2016, 6, 39253.

(37) Barriga, J.; Coto, B.; Fernandez, B. Molecular dynamics study of optimal packing structure of OTS self-assembled monolayers on $\mathrm{SiO}_{2}$ surfaces. Tribol. Int. 2007, 40, $960-966$.

(38) Mar, W.; Klein, M. L. Molecular dynamics study of the self-assembled monolayer composed of $\mathrm{S}\left(\mathrm{CH}_{2}\right)_{14} \mathrm{CH}_{3}$ molecules using an all-atoms model. Langmuir 1994, 10, 188196.

(39) Chen, D.; Huang, F.; Cheng, Y.-B.; Caruso, R. A. Mesoporous anatase $\mathrm{TiO}_{2}$ beads with high surface areas and controllable pore sizes: a superior candidate for high-performance dye-sensitized solar cells. Adv. Mater. 2009, 21, 2206-2210.

(40) Ko, K. H.; Lee, Y. C.; Jung, Y. J. Enhanced efficiency of dye-sensitized $\mathrm{TiO}_{2}$ solar cells (DSSC) by doping of metal ions. J. Colloid Interface Sci. 2005, 283, 482 - 487. 
(41) Yu, J.; Fan, J.; Lv, K. Anatase $\mathrm{TiO}_{2}$ nanosheets with exposed (001) facets: improved photoelectric conversion efficiency in dye-sensitized solar cells. Nanoscale 2010, 2, $2144-2149$.

(42) Raja, S.; Satheeshkumar, C.; Rajakumar, P.; Ganesan, S.; Maruthamuthu, P. Influence of triazole dendritic additives in electrolytes on dye-sensitized solar cell (DSSC) performance. J. Mater. Chem. 2011, 21, 7700-7704.

(43) Zuleta, M.; Yu, S.; Ahmadi, S.; Boschloo, G.; Gothelid, M.; Hagfeldt, A. Monitoring N719 dye configurations on $(1 \times n)$-reconstructed anatase (100) by means of STM: reversible configurational changes upon illumination. Langmuir 2010, 26, 13236-13244.

(44) Al-Abadleh, H. A.; Voges, A. B.; Bertin, P. A.; Nguyen, S. T.; Geiger, F. M. Chromium (VI) binding to functionalized silica/water interfaces studied by nonlinear optical spectroscopy. J. Am. Chem. Soc. 2004, 126, 11126-11127.

(45) Chen, E. H.; Walter, S. R.; Nguyen, S. T.; Geiger, F. M. Arylsilanated $\mathrm{SiO}_{x}$ surfaces for mild and simple two-step click functionalization with small molecules and oligonucleotides. J. Phys. Chem. C 2012, 116, 19886-19892.

(46) Hung, K.-K.; Stege, U.; Hore, D. K. IR absorption, raman scattering, and IR-vis sumfrequency generation spectroscopy as quantitative probes of surface structure. App. Spectrosc. Rev. 2015, 50, 351-376.

(47) Wang, C.-y.; Groenzin, H.; Shultz, M. J. Comparative study of acetic acid, methanol, and water adsorbed on anatase $\mathrm{TiO}_{2}$ probed by sum frequency generation spectroscopy. J. Am. Chem. Soc. 2005, 127, 9736-9744.

(48) Vanselous, H.; Stingel, A. M.; Petersen, P. B. Interferometric 2D sum frequency generation spectroscopy reveals structural heterogeneity of catalytic monolayers on transparent materials. J. Phys. Chem. Lett. 2017, 8, 825-830. 
(49) Schleeger, M.; Nagata, Y.; Bonn, M. Quantifying surfactant alkyl chain orientation and conformational order from sum frequency generation spectra of $\mathrm{CH}$ modes at the surfactant-water interface. Phys. Chem. Lett. 2014, 5, 3737-3741.

(50) Luber, S. Sum frequency generation of acetonitrile on a rutile (110) surface from density functional theory-based molecular dynamics. J. Phys. Chem. Lett. 2016, 7, 5183-5187.

(51) Sengupta, S.; Bromley III, L.; Velarde, L. Aggregated states of chalcogenorhodamine dyes on nanocrystalline titania revealed by doubly resonant sum frequency spectroscopy. J. Phys. Chem. C 2017, 121, 3424-3436.

(52) Ge, A.; Rudshteyn, B.; Psciuk, B. T.; Xiao, D.; Song, J.; Anfuso, C. L.; Ricks, A. M.; Batista, V. S.; Lian, T. Surface-induced anisotropic binding of a Rhenium $\mathrm{CO}_{2^{-}}$ reduction catalyst on Rutile $\mathrm{TiO}_{2}$ (110) surfaces. J. Phys. Chem. C 2016, 120, 2097020977.

(53) Ye, S.; Kathiravan, A.; Hayashi, H.; Tong, Y.; Infahsaeng, Y.; Chabera, P.; Pascher, T.; Yartsev, A.; Isoda, S.; Imahori, H. et al. Role of adsorption structures of Zn-porphyrin on $\mathrm{TiO}_{2}$ in dye-sensitized solar cells studied by sum frequency generation vibrational spectroscopy and ultrafast spectroscopy. J. Phys. Chem. C 2013, 117, 6066-6080.

(54) Clark, M. L.; Rudshteyn, B.; Ge, A.; Chabolla, S. A.; Machan, C. W.; Psciuk, B. T.; Song, J.; Canzi, G.; Lian, T.; Batista, V. S. et al. Orientation of cyano-substituted bipyridine $\operatorname{Re}(\mathrm{I})$ fac-Tricarbonyl electrocatalysts bound to conducting Au surfaces. $J$. Phys. Chem. C 2016, 120, 1657-1665.

(55) Anfuso, C. L.; Snoeberger III, R. C.; Ricks, A. M.; Liu, W.; Xiao, D.; Batista, V. S.; Lian, T. Covalent attachment of a rhenium bipyridyl $\mathrm{CO}_{2}$ reduction catalyst to rutile $\mathrm{TiO}_{2}$. J. Am. Chem. Soc. 2011, 133, 6922-6925.

(56) Urza-Leiva, R. A.; Rampino, S.; Arratia-Perez, R.; Mosconi, E.; Pastore, M.; De Angelis, F. Thermal fluctuations on Frster resonance energy transfer in dyadic solar cell 
sensitizers: a combined ab-initio molecular dynamics and TDDFT investigation. $J$. Phys. Chem. C 2015, 119, 16490-16499.

(57) Gebauer, R.; De Angelis, F. A combined molecular dynamics and computational spectroscopy study of a dye-sensitized solar cell. New J. Phys. 2011, 13, 085013.

(58) Feng, S.; Li, Q.-S.; Sun, P.-P.; Niehaus, T. A.; Li, Z.-S. Dynamic characteristics of aggregation effects of organic dyes in dye-sensitized solar cells. ACS Appl. Mater. Interfaces 2015, 7, 22504-22514.

(59) Ding, W.-L.; Li, Q.-S.; Li, Z.-S. Anti-aggregation and intra-type Förster resonance energy transfer in bulky indoline sensitizers for dye-sensitized solar cells: a combined DFT/TDDFT and molecular dynamics study. J. Mater. Chem. A 2015, 3, 1994819959.

(60) Christianson, J. R.; Schmidt, J. Structural heterogeneity and dynamics of dyes on $\mathrm{TiO}_{2}$ : implications for charge transfer across organic-inorganic interfaces. Phys. Chem. Chem. Phys. 2015, 17, 3731-3740.

(61) Maggio, E.; Troisi, A. An expression for the bridge-mediated electron transfer rate in dye-sensitized solar cells. Phil. Trans. R. Soc. A 2014, 372, 20130011.

(62) Maggio, E.; Martsinovich, N.; Troisi, A. Evaluating charge recombination rate in dyesensitized solar cells from electronic structure calculations. J. Phys. Chem. C 2012, $116,7638-7649$.

(63) Buscher, C. T.; McBranch, D.; Li, D. Understanding the relationship between surface coverage and molecular orientation in polar self-assembled monolayers. J. Am. Chem.Soc. 1996, 118, 2950-2953.

(64) Chen, C.; Liao, J.-Y.; Chi, Z.; Xu, B.; Zhang, X.; Kuang, D.-B.; Zhang, Y.; Liu, S.; $\mathrm{Xu}$, J. Metal-free organic dyes derived from triphenylethylene for dye-sensitized solar 
cells: tuning of the performance by phenothiazine and carbazole. J. Mater. Chem. 2012, 22, 8994-9005.

(65) Matsui, M.; Ito, A.; Kotani, M.; Kubota, Y.; Funabiki, K.; Jin, J.; Yoshida, T.; Minoura, H.; Miura, H. The use of indoline dyes in a zinc oxide dye-sensitized solar cell. Dyes Pigm. 2009, 80, 233 - 238.

(66) Hutter, J.; Iannuzzi, M.; Schiffmann, F.; VandeVondele, J. CP2K: atomistic simulations of condensed matter systems. WIREs Comput. Mol. Sci. 2014, 4, 15-25.

(67) Perdew, J. P.; Burke, K.; Ernzerhof, M. Generalized gradient approximation made simple. Phys. Rev. Lett. 1996, 77, 3865-3868.

(68) VandeVondele, J.; Hutter, J. Gaussian basis sets for accurate calculations on molecular systems in gas and condensed phases. J. Chem. Phys. 2007, 127, 114105.

(69) Grimme, S.; Antony, J.; Ehrlich, S.; Krieg, H. A consistent and accurate ab initio parametrization of density functional dispersion correction (DFT-D) for the 94 elements H-Pu. J. Chem. Phys. 2010, 132, 154104.

(70) Monti, S.; Pastore, M.; Li, C.; De Angelis, F.; Carravetta, V. Theoretical investigation of adsorption, dynamics, self-aggregation, and spectroscopic properties of the D102 indoline dye on an anatase (101) substrate. J. Phys. Chem. C 2016, 120, 2787-2796. 


\section{Graphical TOC Entry}

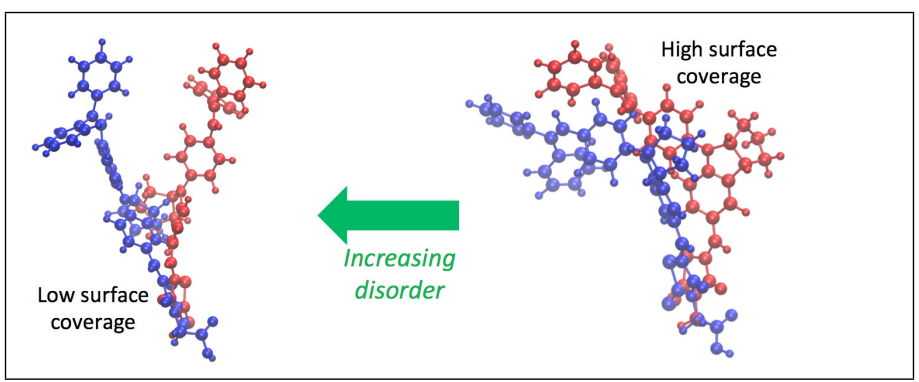

